\title{
Myocardial infarction in the early postoperative period of renal transplantation: Case report and review of literature
}

\author{
Ahmet Karabulut* \\ Department of Cardiology, AcibademAtakent Hospital, Acıbadem University School of Medicine, Halkah Merkez Mah. Turgut Özal Bulvar, No:16, 34303, Kucukcekmece, Istanbul, Turkey
}

\section{A R T I C L E I N F O}

\section{Article history:}

Received 23 March 2016

Received in revised form 13 April 2016

Accepted 14 April 2016

Available online 23 April 2016

\section{Keywords:}

Myocardial infarction

End-stage renal disease

Transplantation

\begin{abstract}
A B S T R A C T
Coronary artery disease (CAD) is the leading cause of mortality in the end-stage renal disease. Although risk of myocardial infarction (MI) decreases after renal transplantation compared to hemodialysis period, CAD remain the major cause of mortality after transplantation also. Risk of MI is maximal during the perioperative period and it's incidence decreases gradually after transplantation. Pre-operative cardiac evaluation is essential prior to renal transplantion. Herein, we prensented a case of acute anterior myocardial infarction observed in the early post-operative period of renal transplantation and review the current literature. We discussed the demographic and clinical predictors of the perioperative cardiac events.

(C) 2016 The Society of Cardiovascular Academy. Production and hosting by Elsevier B.V. All rights reserved. This is an open access article under the CC BY-NC-ND license (http://creativecommons.org/licenses/by-nc-nd/4.0/).
\end{abstract}

\section{Introduction}

Coronary artery disease (CAD) is the leading cause of mortality in end-stage renal disease (ESRD), hence ESRD is accepted as the equivalent of CAD. Although risk of myocardial infarction (MI) decreases after renal transplantation compared to the hemodialysis period, CAD remains the major clinical issue regarding mortality. ${ }^{1}$ Risk of MI is at peak during the perioperative period and its incidence decreases gradually after transplantation. ${ }^{1}$ Effective preoperative cardiac evaluation prior to renal transplantation may prevent the possible cardiac complication in the perioperative period. Herein, we presented a case of acute anterior MI observed in the early postoperative transplantation period and discussed the perioperative risk assessment and management.

\section{Case report}

A 57-year-old male patient was admitted to hospital for renal transplantation. On medical background, he had been followed up with a diagnosis of polycystic kidney disease and he was taking hemodialysis therapy for 10 years. Coronary bare metal stent $(3.0 \times 20 \mathrm{~mm})$ was implanted to the proximal left anterior descending artery (LAD) a year ago and he was free from anginal symptoms for a year. He was a current smoker and also under medication for hypertension. Physical examination did not show specific pathology and functional capacity was more than 7 METS. He also did not define typical anginal chest pain on exertion. Laboratory data showed dyslipidemia with LDL cholesterol $150 \mathrm{mg} / \mathrm{dl}$. Aspirin therapy was discontinued for a week and the patient

\footnotetext{
* Tel.: + 90 5053577477; fax: +902124044445.

E-mail address: drkarabulut@yahoo.com.

Peer review under responsibility of The Society of Cardiovascular Academy.
}

did not take any additional antiagregant therapy. Live donor transplantation was performed without any complication. In the postoperative period, the patient defined atypical chest pain which was not thought as an acute cardiac event. But, there was not any ischemic finding in the ECG. The patient was discharged from the hospital on the seventh postoperative day. However, the patient was readmitted to hospital on the same day with a complaint of typical chest pain, palpitation, and shortness of breath. ECG showed anterior ST elevation and the patient was transferred to a catheter laboratory for primary coronary interventions. Coronary angiography revealed thrombotic critical lesions in the mid and distal LAD (Fig. 1A). Angioplasty and stent deployment was performed to LAD lesion (Fig. 1B). Bare metal stent was preferred by the operator to shorten the dual antiplatelet therapy. Heparin 60 unit/ $\mathrm{kg}$ was given prior to angioplasty and was not continued after procedure. The patient was discharged without any complication including bleeding and additional renal pathology. Dual antiplatelet therapy consists of acetylsalicylic acid $100 \mathrm{mg}$ and clopidogrel $75 \mathrm{mg}$ daily was prescribed for a month. One-year follow-up of the patient was uneventful with functional renal graft.

\section{Discussion}

Kidney transplantation is associated with $17 \%$ risk reduction for myocardial infarction (MI) compared to patients in the waiting list. ${ }^{1}$ However, MI remains a leading cause of death in patients with functioning renal transplantation. The risk of MI is highest in the early postoperative period and mortality rates after hospitalization for MI is approximately $50 \%$ at 5 years, which affects allograft longevity also. ${ }^{1}$ Perioperative cardiac events might occur as a result of demandmediated ischemia and/or plaque rupture. A detailed preoperative cardiac assessment should be performed to cover both clinical issues. ${ }^{2}$ 


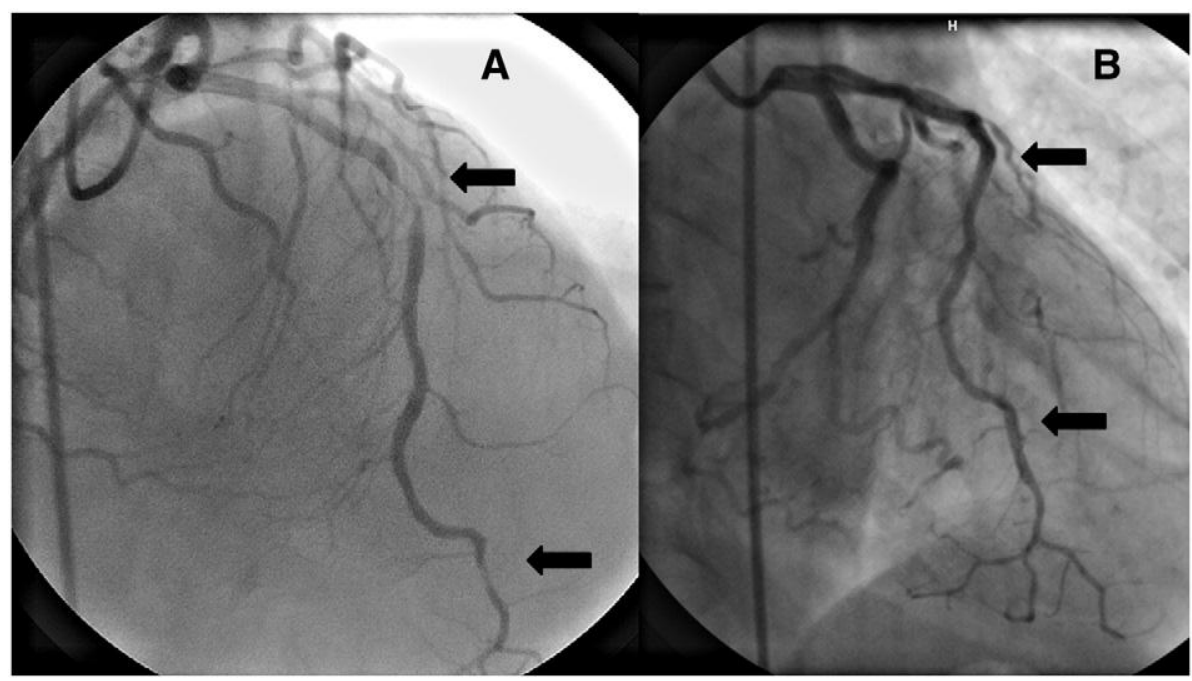

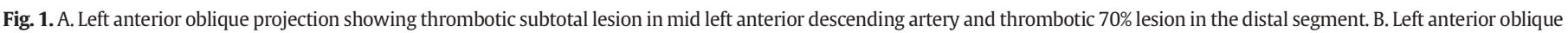
projection showing successful percutaneous coronary interventions.

The risk and predictors of post-renal transplantation MI are not well described. Nonetheless, renal allograft recipients have higher prevalence of traditional atherosclerotic risk factors. The risk factors for posttransplantation period might be defined as age $>60$,obesity, smoking, left ventricular hypertrophy, diabetes, hypertension, and dyslipidemia. ${ }^{2-3}$ Besides traditional risk factors, male gender, time on dialysis before transplantation (>a year), donor history of hypertension, transplantation from older donor, immunosuppressive regimen, quality of allograft function, and posttransplantation diabetes also are proposed as predictors of cardiovascular risk in this population. ${ }^{3-4}$ Both Framingham risk score and European Society of Cardiology Score chart can be used to determine risk for cardiovascular disease especially in low-risk patients. Framingham risk score may underestimate cardiovascular risk in the high-risk patients. ${ }^{5} \mathrm{CT}$ coronary angiography has a high negative predictive value in screening of CAD and it would be an ideal screening test in low- to intermediate-risk patients. However, coronary calcification is usually widespread in the ESRD, which may interfere with the sensitivity and specificity of the study. ${ }^{2}$ Myocardial perfusion scintigraphy or dobutamine stress echocardiography is the primarily recommended noninvasive tests for asymptomatic high-risk patients. Despite, both studies may give useful information in the assessment of coronary ischemia mediated by demand-blood supply discrepancy, they would not predict cardiac events secondary to plaque rupture. ${ }^{2}$ Thus, modification of risk factors and effective medical therapy prior to surgery may diminish the acute thrombotic ischemic events. In addition, preoperative coronary angiography might be a logical approach for assessing cardiovascular risk in symptomatic high-risk patients. Our case was already diagnosed CAD and also possess traditional risk factors including hypertension, dyslipidemia, and smoking. Relatively longer dialysis time and immunosuppressive therapy are the other risk factors for cardiac complications. Preoperative coronary angiography would be logical to show patency of stent regarding our case. On contrary, preoperative angiography would not predict the acute coronary events for our patients. Because LAD stent was already patent and preoperative angiography would not change the transplantation schedule, efficacy of preoperative cardiac revascularization was also not clear. Lifestyle modification including cessation of smoking and regular exercise and also regulation of medical antiischemic agents would be the most important prognostic factors to prevent perioperative coronary events for our patient. On the other hand, postoperative routine screening of troponin I levels may also be beneficial for diagnosing cardiac complication. Although abnormal troponin level is highly prevalent following renal transplantation, normal troponin levels had a high negative predictive value in excluding patients likely to develop postoperative MI. ${ }^{6}$ Troponin level was not checked in our patients despite atypical chest pain definition.

Early postoperative percutaneous coronary intervention is associated with bleeding complication because of dual antiplatelet therapy plus heparin. It was reported that thrombus aspiration and lone balloon angioplasty may be logical in the selected cases. ${ }^{7}$ However, our case characteristic was not suitable for both strategy because of tight thrombotic subtotal lesion in the mid LAD. The operator preferred bare metal stent to shorten dual antiplatelet therapy. Bare metal stents are the classical choice in the intervention before the transplantation surgery for shorter dual antiplatelet therapy. A small case series show that, kidney transplant surgery may performed under antiplatelet therapy with lower bleeding complication contrary to general consensus of discontinuation of antiplatelet therapy for 5 days. ${ }^{2,8}$ Drug eluting stents (DES) are superior to BMS in terms of restenosis and acute coronary events also in the patients with ESRD. Compared to older DES, new-generation DES showed less stent thrombosis with shorter dual antiplatelet therapy. ${ }^{9}$ Palmerini $\mathrm{T}$ et al demonstrated that major adverse cardiac events were similar with the 3-month and 12-month antiplatelet therapy in the new-generation DES era. ${ }^{10}$ We think that dual antiplatelet therapy is not a limitation for renal transplantation recipients and DES should replace traditional BMS implantation in ESRD patients regardless of the time of transplantation.

\section{Conclusion}

Acute myocardial infarction is a major cause of mortality in the perioperative period of renal transplantation. A detailed preoperative assessment of cardiac status, effective preoperative anti-ischemic therapy including lifestyle management and preoperative coronary angiography in the symptomatic high-risk patients, will prevent mortality and provide allograft longevity.

\section{Acknowledgments}

The authors did not report conflict of interest regarding this work. This work was not supported by any company.

\section{References}

1. Kasiske BL, Maclean JR, Snyder JJ. Acute myocardial infarction and kidney transplantation. J Am Soc Nephrol 2006;17(3):900-907 (Mar). 
2. Lentine KL, Costa SP, Weir MR, et al. Cardiac disease evaluation and management among kidney and liver transplantation candidates: a scientific statement from American Heart Association and the American College of Cardiology Foundation. J Am Coll Cardiol 2012;60:434-480 (Jul 31).

3. Lentine KL, Brennan DC, Schnitzler MA. Incidence and predictors of myocardial infarction after kidney transplantation. J Am Soc Nephrol 2005;16(2):496-506 (Feb).

4. Fazelzadeh A, Mehdizadeh A, Ostovan MA, Raiss-Jalali GA. Incidence of cardiovascular risk factors and complications before and after kidney transplantation. Transplant Proc 2006;38(2):506-508 (Mar).

5. Ducloux D, Kazory A, Chalopin JM. Predicting coronary heart disease in renal transplant recipients: a prospective study. Kidney Int 2004;66:441-447.

6. Shroff GR, Akkina SK, Miedema MD, Madlon-Kay R, Herzog CA, Kasiske BL. Troponin I levels and postoperative myocardial infarction following renal transplantation. Am J Nephrol 2012;35(2):175-180.
7. Karabulut A. Thrombus aspiration may decrease bleeding risk in the early postoperative myocardial infarction treated with percutaneous intervention. Anadolu Kardiyol Derg 2013;13(4):402 (Jun)

8. Roik M, Wretowski D, Labyk A, et al. Acute ST segment elevation myocardial infarction treated with delayed angioplasty in a patient with anomalous origin of the right coronary artery in the early phase after kidney transplantation. Postep Kardiol Inter 2014;10:3179.

9. Summaria F, Giannico MB, Talarico GP, Patrizi R. Percutaneous coronary interventions and antiplatelet therapy in renal transplant recipients. Ther Adv Cardiovasc Dis 2016;10:86-97.

10. Palmerini T, Sangiorgi D, Valgimigli M, et al. Short- versus long-term dual antiplatelet therapy after drug-eluting stent implantation: an individual patient data pairwise and network meta-analysis. J Am Coll Cardiol 2015;65:1092-1102. 